\title{
Fast separation in a graph with an excluded minor $^{\dagger}$
}

\author{
Bruce Reed $^{1}$ and David R. Wood ${ }^{2}$ \\ ${ }^{1}$ School of Computer Science, McGill University, Montréal, Canada (breedecs . mcgill . ca) \\ ${ }^{2}$ Departament de Matemàtica Aplicada II, Universitat Politècnica de Catalunya, Barcelona, Spain \\ (david.wood@upc.edu)
}

\begin{abstract}
Let $G$ be an $n$-vertex $m$-edge graph with weighted vertices. A pair of vertex sets $A, B \subseteq V(G)$ is a $\frac{2}{3}$-separation of order $|A \cap B|$ if $A \cup B=V(G)$, there is no edge between $A \backslash B$ and $B \backslash A$, and both $A \backslash B$ and $B \backslash A$ have weight at most $\frac{2}{3}$ the total weight of $G$. Let $\ell \in \mathbb{Z}^{+}$be fixed. Alon, Seymour and Thomas [J. Amer. Math. Soc. 1990] presented an algorithm that in $\mathcal{O}\left(n^{1 / 2} m\right)$ time, either outputs a $K_{\ell}$-minor of $G$, or a separation of $G$ of order $\mathcal{O}\left(n^{1 / 2}\right)$. Whether there is a $\mathcal{O}(n+m)$ time algorithm for this theorem was left as open problem. In this paper, we obtain a $\mathcal{O}(n+m)$ time algorithm at the expense of $\mathcal{O}\left(n^{2 / 3}\right)$ separator. Moreover, our algorithm exhibits a tradeoff between running time and the order of the separator. In particular, for any given $\epsilon \in\left[0, \frac{1}{2}\right]$, our algorithm either outputs a $K_{\ell}$-minor of $G$, or a separation of $G$ with order $\mathcal{O}\left(n^{(2-\epsilon) / 3}\right)$ in $\mathcal{O}\left(n^{1+\epsilon}+m\right)$ time.
\end{abstract}

Keywords: graph algorithm, separator, minor

\section{Introduction}

We consider graphs $G$ that are simple, finite, and undirected. Let $V(G)$ and $E(G)$ denote the vertex and edge sets of $G$. Let $|G|:=|V(G)|$ and $\|G\|:=|E(G)|$. A separation of $G$ is a pair $\{A, B\}$ of vertex sets $A, B \subseteq V(G)$ such that $A \cup B=V(G)$, and there is no edge with one endpoint in $A \backslash B$ and the other endpoint in $B \backslash A$. The order of $\{A, B\}$ is $|A \cap B|$. The set $A \cap B$ is called a separator of $G$. A weighting of $G$ is a function $w: V(G) \rightarrow \mathbb{R}^{+}$. Let $w(S):=\sum_{v \in S} w(v)$ for all $S \subseteq V(G)$, and $w(G):=w(V(G))$. We say $(G, w)$ is a weighted graph. A separation $\{A, B\}$ of a weighted graph $(G, w)$ is an $\alpha$-separation if $w(A \backslash B) \leq \alpha \cdot w(G)$ and $w(B \backslash A) \leq \alpha \cdot w(G)$.

A 'separator theorem' is of the format: for some $0<\alpha<1$ and $0<\epsilon \leq 1$, every graph $G$ from a certain family has an $\alpha$-separation of $\operatorname{order} \mathcal{O}\left(|G|^{1-\epsilon}\right)$. Applications of separator theorems are numerous, and include VLSI circuit layout, approximation algorithms using the divide-and-conquer paradigm, solving sparse systems of linear equations, pebbling games, and graph drawing. See the recent monograph by Rosenberg and Heath [9] for more details.

A seminal theorem due to Lipton and Tarjan [5] states that every weighted planar graph $G$ has a $\frac{2}{3}$ separation of order $\mathcal{O}\left(|G|^{1 / 2}\right)$ that can be computed in $\mathcal{O}(|G|+\|G\|)$ time. This result was generalised

\footnotetext{
${ }^{\dagger}$ Research of B.R. is supported by NSERC. Research of D.W. is supported by the Government of Spain grant MEC SB2003-0270. 1365-8050 (C) 2005 Discrete Mathematics and Theoretical Computer Science (DMTCS), Nancy, France
} 
for graphs with an excluded minor by Alon et al. [1] (see [2, 3, 7] for related results). A graph $H$ is a minor of a graph $G$ if $H$ can be obtained from a subgraph of $G$ by contracting edges, in which case we say that $G$ has an $H$-minor. The Kuratowski-Wagner Theorem states that a graph is planar if and only if it has no $K_{5}$-minor and no $K_{3,3}$-minor. An $H$-model in $G$ is a set of disjoint connected subgraphs $\left\{X_{v}: v \in V(H)\right\}$ indexed by the vertices of $H$, such that for every edge $v w \in E(H)$, there is an edge $x y \in E(G)$ with $x \in X_{v}$ and $y \in X_{w}$. Clearly $G$ has an $H$-minor if and only if $G$ has an $H$-model. We choose to work with $H$-models rather than $H$-minors.

Theorem 1 (Alon et al. [1]) There is an algorithm with running time $\mathcal{O}\left((\ell \cdot|G|)^{1 / 2} \cdot(|G|+\|G\|)\right)$ that, given $\ell \in \mathbb{Z}^{+}$and a weighted graph $(G, w)$, either outputs:

(a) a $K_{\ell}$-model of $G$, or

(b) a $\frac{2}{3}$-separation of $(G, w)$ of order at most $\ell^{3 / 2} \cdot|G|^{1 / 2}$.

Suppose that $\ell$ is fixed. It follows from a result of Mader [6] (see Theorem 3) that Theorem 1 can be implemented in $\mathcal{O}\left(|G|^{3 / 2}+\|G\|\right)$ time. Alon et al. [1] left as an open problem whether linear time is possible. The main result of this paper is the following partial answer to this question. We obtain a linear running time at the expense of a slightly larger separator (and a larger dependence on $\ell$ ). Moreover, our algorithm exhibits a tradeoff between running time (ranging from $\mathcal{O}(n)$ to $\mathcal{O}\left(n^{3 / 2}\right)$ ) and the order of the separator (ranging from $\mathcal{O}\left(n^{2 / 3}\right)$ to $\mathcal{O}\left(n^{1 / 2}\right)$ ).

Theorem 2 There is an algorithm with running time $\mathcal{O}\left(2^{\left(3 \ell^{2}+7 \ell-3\right) / 2} \cdot|G|^{1+\epsilon}+\ell \cdot\|G\|\right)$ that, given $\epsilon \in\left[0, \frac{1}{2}\right], \ell \in \mathbb{Z}^{+}$, and a weighted graph $(G, w)$, either outputs:

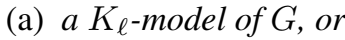

(b) a $\frac{2}{3}$-separation of $(G, w)$ of order at most $2^{\left(\ell^{2}+3 \ell+1\right) / 2} \cdot|G|^{(2-\epsilon) / 3}$.

Note that for applications to divide-and-conquer algorithms a separation of order $\mathcal{O}\left(|G|^{1-\epsilon}\right)$, for some constant $\epsilon>0$, is all that is needed.

The idea behind the proof of Theorem 2 is simple. We now outline the proof for fixed $\ell$ and with $\epsilon=0$. Suppose that in $\mathcal{O}(|G|+\|G\|)$ time, we can find a partition of $V(G)$ into $|G|^{2 / 3}$ connected subgraphs $\left\{S_{1}, S_{2}, \ldots, S_{|G|^{2 / 3}}\right\}$, each containing $\mathcal{O}\left(|G|^{1 / 3}\right)$ vertices. Let $H$ be the weighted graph obtained from $G$ by contracting each $S_{i}$ to a vertex $v_{i}$ with weight $w\left(v_{i}\right)=w\left(S_{i}\right)$. Then apply Theorem 1 to $H$ to either obtain a $K_{\ell}$-model in $H$ which defines a $K_{\ell}$-model in $G$, or a $\frac{2}{3}$-separation $\{A, B\}$ of $H$ with order $\mathcal{O}\left(|H|^{1 / 2}\right)=\mathcal{O}\left(|G|^{1 / 3}\right)$, in which case $\left\{\bigcup\left\{S_{i}: v_{i} \in A\right\}, \bigcup\left\{S_{i}: v_{i} \in B\right\}\right\}$ a is a $\frac{2}{3}$-separation of $G$ with order $\mathcal{O}\left(|G|^{2 / 3}\right)$. The running time is $\mathcal{O}\left(|H|^{3 / 2}+\|H\|\right) \subseteq \mathcal{O}(|G|+\|G\|)$. The proof of Theorem 2 is actually a little different from this outline. In particular, the subgraphs $S_{i}$ will not necessarily be connected, but we will still be able to convert the output from Theorem 1 applied to $H$ to the desired output for $G$. By relaxing the connectivity condition, we are able to prove that an appropriate partition exists.

We will use the following notation for a graph $G$. For $x \in V(G)$, let $N(x):=\{y \in V(G): x y \in$ $E(G)\}$. For a subgraph $X$ of $G$, let $N(X):=\bigcup\{N(x) \backslash X: x \in X\}$. Where there is no confusion, a set of vertices $S \subseteq V(G)$ will also refer to the subgraph of $G$ induced by $S$.

\section{Mader's Theorem}

This section contains a number of easily proved results - see the full version of the paper for details. We start with an algorithmic version of a theorem of Mader [6] (cf. [8]). 


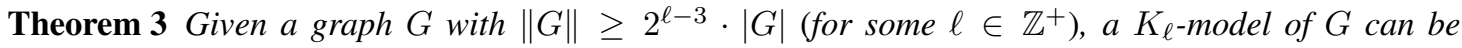
computed in $\mathcal{O}(\ell(|G|+\|G\|))$ time.

Note that if we ignore the running time, Theorem 3 is far from best possible. Kostochka [4] and Thomason [10] independently proved that if $\|G\| \in \Omega(\ell \sqrt{\log \ell} \cdot|G|)$ then $G$ has a $K_{\ell}$-model. Theorem 3 implies the following slightly faster version of Theorem 1 (for fixed $\ell$ )

Theorem 4 There is an algorithm with running time $\mathcal{O}\left(2^{2 \ell} \cdot|G|^{3 / 2}+\ell \cdot\|G\|\right)$ that, given $\ell \in \mathbb{Z}^{+}$and a weighted graph $(G, w)$, either outputs:

(a) a $K_{\ell}$-model of $G$, or

(b) a $\frac{2}{3}$-separation of $(G, w)$ of order at most $\ell^{3 / 2} \cdot|G|^{1 / 2}$.

A $k$-clique of $G$ is a (not necessarily maximal) set of $k$ pairwise adjacent vertices of $G$. If every subgraph of $G$ has a vertex of degree at most $d$, then $G$ is $d$-degenerate. For example, Theorem 3 implies that a graph with no $K_{\ell}$-minor is $2^{\ell-2}$-degenerate. It is easily proved that a $d$-degenerate graph $G$ with no $k$-clique has less than $d^{k-1} \cdot|G|$ cliques. Hence a graph $G$ with no $K_{\ell}$-minor has less than $2^{(\ell-2)(\ell-1)} \cdot|G|$ cliques. For an algorithm, we have the following result.

Lemma 1 Given a graph $G$ with no $k$-clique and at least $2^{(\ell-2)(k-1)} \cdot|G|$ cliques (for some $\left.\ell \in \mathbb{Z}^{+}\right)$, a $K_{\ell}$-minor of $G$ can be computed in $\mathcal{O}(\ell(|G|+\|G\|))$ time.

\section{Proof of Theorem 2}

Let $G$ be a graph. Let $\mathcal{A}$ be a partition of $V(G)$. Let $H$ be the graph obtained from $G$ by collapsing each part $S \in \mathcal{A}$ to a single vertex $v$, and replacing parallel edges by a single edge. Denote $H_{v}:=S$. We say $\left\{H_{v}: v \in V(H)\right\}$ is an $H$-partition of $G$. Furthermore, $\left\{H_{v}: v \in V(H)\right\}$ is a connected H-partition of $G$ if $v w \in E(H)$ if and only if there is an edge of $G$ between every component of $H_{v}$ and every component of $H_{w}$. We prove the following lemma.

Lemma 2 There is an algorithm with running time $\mathcal{O}\left(2^{2 \ell} \cdot|G|+\|G\|\right)$ that, given $\ell, k \in \mathbb{Z}^{+}$and a graph $G$, outputs a connected $H$-partition of $G$ such that either:

(a) $H$ has a $K_{\ell}$-model (which is also output), or

(b) $|H| \leq 2^{\ell^{2}+\ell-1} \cdot|G| \cdot k^{-1}$, and $\left|H_{x}\right|<2 k$ for all $x \in V(H)$.

Proof of Theorem 2 assuming Lemma 2: Apply Lemma 2 with $k=\left\lfloor|G|^{(1-2 \epsilon) / 3}\right\rfloor$. First suppose that Lemma 2 outputs a $K_{\ell}$-model $\left\{S_{1}, S_{2}, \ldots, S_{\ell}\right\}$ of $H$. Thus each $S_{i}$ is a connected subgraph of $H$. Choose a connected component $Z_{v}$ of $H_{v}$ for each $v \in V(H)$. Let $T_{i}:=\bigcup\left\{Z_{v}: v \in S_{i}\right\}$. Then $\left\{T_{1}, T_{2}, \ldots, T_{\ell}\right\}$ is a $K_{\ell}$-model of $G$.

Otherwise $|H| \leq 2^{\ell^{2}+\ell-1} \cdot|G|^{2(1+\epsilon) / 3}$, and $\left|H_{x}\right|<2|G|^{(1-2 \epsilon) / 3}$ for all $x \in V(H)$. Let $w(v):=w\left(H_{v}\right)$ for all $v \in V(H)$. Apply Theorem 4 to $(H, w)$. The running time is

$\mathcal{O}\left(2^{2 \ell} \cdot|H|^{3 / 2}+\ell \cdot\|H\|\right) \subseteq \mathcal{O}\left(2^{2 \ell} \cdot\left(2^{\ell^{2}+\ell-1} \cdot|G|^{2(1+\epsilon) / 3}\right)^{3 / 2}+\ell \cdot\|G\|\right) \subseteq \mathcal{O}\left(2^{\left(3 \ell^{2}+7 \ell-3\right) / 2} \cdot|G|^{1+\epsilon}+\ell \cdot\|G\|\right)$.

We either obtain a $K_{\ell}$-model of $H$, or a $\frac{2}{3}$-separation of $H$ with order at most $\ell^{3 / 2} \cdot|H|^{1 / 2}$. In the first case, $G$ has a $K_{\ell}$-model as proved above.

Now suppose that we obtain a $\frac{2}{3}$-separation $\{A, B\}$ of $(H, w)$ with order

$$
|A \cap B| \leq \ell^{3 / 2} \cdot|H|^{1 / 2} \leq \ell^{3 / 2} \cdot\left(2^{\ell^{2}+\ell-1}|G|^{2(1+\epsilon) / 3}\right)^{1 / 2} \leq 2^{\left(\ell^{2}+3 \ell-1\right) / 2} \cdot|G|^{(1+\epsilon) / 3} .
$$


Let $X:=\bigcup\left\{H_{v}: v \in A\right\}$ and $Y:=\bigcup\left\{H_{v}: v \in B\right\}$. Then $\{X, Y\}$ is a separation of $G$ with order

$|X \cap Y|=\left|\bigcup\left\{H_{v}: v \in A \cap B\right\}\right| \leq 2^{\left(\ell^{2}+3 \ell-1\right) / 2} \cdot|G|^{(1+\epsilon) / 3} \cdot 2|G|^{(1-2 \epsilon) / 3} \leq 2^{\left(\ell^{2}+3 \ell+1\right) / 2} \cdot|G|^{(2-\epsilon) / 3}$.

We have $w(X \backslash Y)=w(A \backslash B) \leq \frac{2}{3} w(H)=\frac{2}{3} w(G)$. Similarly $w(B \backslash A) \leq \frac{2}{3} w(G)$.

\section{Proof of Lemma 2:}

Step 1: Using a breadth-first search algorithm, compute a maximal set $\mathcal{A}$ of connected subgraphs of $G$ such that $|S|=k$ for all $S \in \mathcal{A}$. Let $\mathcal{B}$ be the set of connected components of $G \backslash \bigcup\{S \in \mathcal{A}\}$. Then $\mathcal{A} \cup \mathcal{B}$ is a partition of $V(G)$, and there is no edge of $G$ between distinct $T_{1}, T_{2} \in \mathcal{B}$. Note that $|T|<k$ for all $T \in \mathcal{B}$, as otherwise $T$ would contain a connected subgraph $X$ with $|X|=k$, which could be added to $\mathcal{A}$.

Step 2: Let $H$ be the graph obtained from $G$ by contracting each set $S \in \mathcal{A} \cup \mathcal{B}$ into a single vertex $v$ with $H_{v}:=S$, and replacing parallel edges by a single edge. Since each $S \in \mathcal{A} \cup \mathcal{B}$ is connected in $G$, $\left\{H_{v}: v \in V(H)\right\}$ is a connected $H$-partition of $G$. Let $A:=\left\{v \in V(H): H_{v} \in \mathcal{A}\right\}$ and $B:=\{v \in$ $\left.V(H): H_{v} \in \mathcal{B}\right\}$. A vertex $v$ of $H$ is big if $\left|H_{v}\right| \geq k$. A vertex $v$ of $H$ is small if $\left|H_{v}\right|<k$. Observe that every vertex in $A$ is big, $B$ is an independent set of $H$, and every vertex in $B$ is small. Partition $B=C \cup D \cup E$, where $C:=\left\{v \in B: \operatorname{deg}_{H}(v) \geq 2^{\ell-2}\right\}, D:=\left\{v \in B: \ell-1 \leq \operatorname{deg}_{H}(v)<2^{\ell-2}\right\}$, and $E:=\left\{v \in B: \operatorname{deg}_{H}(v) \leq \ell-2\right\}$.

Step 3: Suppose that $|C| \geq|A|$. Then the subgraph $C \cup A$ of $H$ has at least $2^{\ell-2} \cdot|C|$ edges and at most $2|C|$ vertices. By Theorem 3, a $K_{\ell}$-model of $C \cup A$ can be computed in $\mathcal{O}(\ell \cdot|G|)$ time. We now assume that $|C|<|A|$.

Step 4: For each vertex $v \in D \cup E$, if there is a pair $x, y \in A$ of distinct neighbours of $v$, such that $\{x, y\}$ has not been assigned any vertex in $D \cup E$, then assign $v$ to $\{x, y\}$. This step can be implemented in $\mathcal{O}\left(2^{2 \ell} \cdot|G|\right)$ time, since each vertex in $D \cup E$ has degree at most $2^{\ell-2}$.

Suppose that there is a vertex $v \in D$ that is not assigned. Let the neighbourhood of $v$ be $\left\{x_{1}, x_{2}, \ldots, x_{d}\right\}$. Then $d \geq \ell-1$. Thus for all $1 \leq i<j \leq d$, there is a distinct vertex $v_{i, j}$ that is assigned to the pair $\left\{x_{i}, x_{j}\right\}$, and $v_{i, j}$ is adjacent to both $x_{i}$ and $x_{j}$. In the graph obtained from $H$ by contracting each edge $x_{i} v_{i, j}$, the subgraph $\left\{x_{1}, x_{2}, \ldots, x_{d}, v\right\}$ is a clique on at least $\ell$ vertices. Thus $H$ has a $K_{\ell}$-model. We now assume that every vertex in $D$ is assigned.

Let $E^{*}$ be the set of assigned vertices in $E$. Consider the graph obtained from $A \cup D \cup E^{*}$ by contracting the edge $v x$ for each $v \in D \cup E^{*}$ assigned to the pair $\{x, y\}$. This graph has $|A|$ vertices and at least $|D|+\left|E^{*}\right|$ edges. Thus if $|D|+\left|E^{*}\right| \geq 2^{\ell-3} \cdot|A|$, then by Theorem $3, H$ has a $K_{\ell}$-model that can be computed in $\mathcal{O}(\ell \cdot|G|)$ time. We now assume that $|D|+\left|E^{*}\right|<2^{\ell-3} \cdot|A|$.

Step 5: Partition $E \backslash E^{*}=\bigcup\left\{P_{1}, P_{2}, \ldots, P_{s}\right\}$ such that for all $u, v \in E \backslash E^{*}$, we have $N(u)=N(v)$ if and only if both $u, v \in P_{i}$ for some $1 \leq i \leq s$. For all $1 \leq i \leq s$, partition $P_{i}=\bigcup\left(P_{i, 1}, P_{i, 2}, \ldots, P_{i, t_{i}}\right)$ such that for all $1 \leq j \leq t_{i}-1, k \leq\left|\bigcup\left\{H_{v}: v \in P_{i, j}\right\}\right|<2 k$, and $\left|\bigcup\left\{H_{v}: v \in P_{i, t_{i}}\right\}\right|<k$. This is possible since $\left|H_{v}\right|<k$ for all $v \in P_{i}$. Collapse each set $P_{i, j}$ into a single vertex $p_{i, j}$ in $H$, whose associated subgraph in $G$ is $H_{p_{i, j}}:=\bigcup\left\{H_{v}: v \in P_{i, j}\right\}$. Since the vertices in $P_{i, j}$ have the same neighbourhood, $\left\{H_{v}: v \in V(H)\right\}$ remains a connected partition of $G$. Let $E_{\text {big }}=\left\{p_{i, j}: 1 \leq i \leq s, 1 \leq\right.$ $\left.j \leq t_{i}-1\right\}$ and $E_{\text {small }}=\left\{p_{i, t_{i}}: 1 \leq i \leq s\right\}$. Then every vertex in $E_{\text {big }}$ is big and every vertex in $E_{\text {small }}$ is small. 
Suppose that $\left|E_{\text {small }}\right| \geq 2^{\ell^{2}} \cdot|A|$. Let $X$ be the graph obtained from $A$ by adding a clique on $N(v)$ for each vertex $v \in E_{\text {small }}$. Since distinct vertices in $E_{\text {small }}$ have distinct neighbourhoods, this process adds at least $\left|E_{\text {small }}\right| \geq 2^{\ell^{2}} \cdot|A|$ cliques. Thus by Lemma 1 , a $K_{\ell}$-model of $X$ can be computed in $\mathcal{O}(|G|)$ time. For every edge $x_{i} x_{j}$ in this $K_{\ell}$-model that is in $X$ but not in $A$, we have $x_{i}, x_{j} \in N(v)$ for some $v \in E_{\text {small }}$. Since $v$ is not assigned, there is a vertex $u \in D \cup E^{*}$ assigned to $\left\{x_{i}, x_{j}\right\}$, and $u$ is adjacent to both $x_{i}$ and $x_{j}$. Since $u$ is not in the $K_{\ell}$-model, we can include $u$ in the connected subgraph of the $K_{\ell}$-model that contains $x_{i}$ or $x_{j}$, and we obtain a $K_{\ell}$-model in $A \cup D \cup E^{*}$ (in particular, without the edge $x_{i} x_{j}$ ). Now assume that $\left|E_{\text {small }}\right|<2^{\ell^{2}} \cdot|A|$.

Step 6: We have now partitioned $V(H)$ into sets $A \cup E_{\text {big }}$ of big vertices, and sets $C \cup D \cup E^{*} \cup E_{\text {small }}$ of small vertices. We have proved that $|C|<|A|,|D|+\left|E^{*}\right|<2^{\ell-3} \cdot|A|$, and $\left|E_{\text {small }}\right|<2^{\ell^{2}} \cdot|A|$. Thus the number of small vertices is less than $\left(1+2^{\ell-3}+2^{\ell^{2}}+1\right) \cdot|A| \leq 2^{\ell^{2}+\ell-2} \cdot|A|$. By definition, the number of big vertices in $H$ is at most $|G| \cdot k^{-1}$. In particular, $|A| \leq|G| \cdot k^{-1}$. Thus $|H| \leq 2^{\ell^{2}+\ell-1} \cdot|G| \cdot k^{-1}$. Moreover, every $\left|H_{v}\right|<2 k$ for every vertex $v \in V(H)$.

\section{References}

[1] Noga Alon, Paul Seymour, and Robin Thomas. A separator theorem for nonplanar graphs. J. Amer. Math. Soc., 3(4):801-808, 1990.

[2] ERic D. Demaine and MohammadTaghi Hajiaghayi. Graphs excluding a fixed minor have grids as large as treewidth, with combinatorial and algorithmic applications through bidimensionality. In Proc. 16th Annual ACM-SIAM Symp. on Discrete Algorithms (SODA '05). ACM, 2005.

[3] Martin Grohe. Local tree-width, excluded minors, and approximation algorithms. Combinatorica, 23(4):613-632, 2003.

[4] Alexandr V. Kostochka. Lower bound of the Hadwiger number of graphs by their average degree. Combinatorica, 4(4):307-316, 1984.

[5] Richard J. Lipton AND Robert E. TARJAn. A separator theorem for planar graphs. SiAM J. Appl. Math., 36(2):177-189, 1979.

[6] Wolfgang Mader. Homomorphieeigenschaften und mittlere Kantendichte von Graphen. Math. Ann., 174:265-268, 1967.

[7] Serge Plotkin, Satish Rao, and Warren D. Smith. Shallow excluded minors and improved graph decompositions. In Proc. 5th Annual ACM-SIAM Symp. on Discrete Algorithms (SODA '94), pp. 462-470. ACM, 1994.

[8] Neil Robertson and Paul D. Seymour. Graph minors. XIII. The disjoint paths problem. $J$. Combin. Theory Ser. B, 63(1):65-110, 1995.

[9] Arnold L. Rosenberg And Lenwood S. Heath. Graph separators, with applications. Frontiers of Computer Science. Kluwer, 2001.

[10] Andrew Thomason. An extremal function for contractions of graphs. Math. Proc. Cambridge Philos. Soc., 95(2):261-265, 1984. 
